\title{
Evolutionary Algorithm Based Optimal Control in Distribution System with Dispersed Generation
}

\author{
P.N.Hrisheekesha \\ Department of $E$ \& $E$ \\ Manipal Institute of Technology \\ Manipal University, Manipal
}

\author{
Dr. Jaydev Sharma \\ Professor \\ Department of Electrical Engg., \\ Indian Institute of Technology Roorkee
}

\begin{abstract}
In this paper, one of the evolutionary algorithm based method, Non-Dominated Sorting Genetic Algorithm (NSGA) has been presented for the Volt / Var control in power distribution systems with dispersed generation (DG). The proposed method is better suited for volt/var control problems. Genetic algorithm approach is used due to its broad applicability, ease of use and high accuracy. A multi-objective optimization problem has been formulated for the volt/var control of the distribution system. The non-dominated sorting genetic algorithm based method proposed in this paper, alleviates the problem of tuning the weighting factors required in solving the multi-objective volt/var control optimization problems. Based on the simulation studies carried out on the distribution system, the proposed scheme has been found to be simple, accurate and easy to apply to solve the multiobjective volt/var control optimization problem of the distribution system with dispersed generation.
\end{abstract}

\section{Keywords}

Dispersed Generation, Distribution System, Evolutionary Algorithm, Non-Dominated Sorting Genetic Algorithm, Voltage / Reactive power control.

\section{INTRODUCTION}

The size and complexity of the modern day power system have increased significantly due to the ever increasing demand of electric power. Hence to supply the increasing demand and to maintain the customer satisfaction, alternate techniques have to be considered by the power supply companies. In recent days the increasing concerns over the environmental issues demand the search for sustainable sources of energy. In this direction the distributed generation (DG) which is both environmental friendly and also can solve the problem of serving the increasing demand of power plays a very important role. The distributed generation consists of small generators in the capacity ranging from $10 \mathrm{~kW}$ to $20 \mathrm{MW}$ (even up to $50 \mathrm{MW}$ ) in a distribution system which are scattered throughout a power system to provide electric power needed by consumers, thereby displacing the need to build additional or upgrade local distribution lines as well as improving the system performance. Dispersed generation is a

subset of DG, with the capacity ranging from 10 to $250 \mathrm{~kW}$, which is connected to the distribution network up to 11 or 110 or
$132 \mathrm{kV}$. This also permits the electricity consumer who is generating electricity for their own needs and to send their surplus electric power into the power grid. Therefore it is a matter of significant concern to consider the integration of distributed generation to the power distribution system.

Recently, due to the complicated environment of the distribution networks under deregulated and competitive power markets, volt/var control has become one of the important tasks in the distribution system planning, operation and control. Distributed generation can have significant impact on voltages, load demand, power losses, system reliability and economy in the distribution networks. Introduction of distributed generation into the distribution system causes reverse power flow and makes the voltage profile complicated[1],[4]. In order to ensure the quality of supply, distribution control center operators impose limits on the allowable voltage levels throughout the network. Since volt/var control is directly related to the power quality of the distribution network and its economy, it is of primary concern to consider in this research work.

Distribution system voltages if within the ANSI C84.1-1995 limits need less attention, but when they exceed the prescribed limits proper means must be provided to control the voltage so as to bring them within the limits [1]-[3]. In the literature many voltage regulation methods are discussed. Kim and Kim [5] addressed the voltage regulation coordination method of DGs in a power distribution system by controlling the reactive power of the DGs. Gonen [6] illustrated LDC voltage regulation method. TaeEung Kim and Jae-Eon Kim [7] developed correlations between the load tap changing transformer (LCT), line drop compensator (LDC) and the output of DG. Joon-Ho Choi and Jae-Chul Kim [8] presented multiple line drop compensation voltage regulation method considering dispersed storage generation systems and unbalanced load diversity. Nigel C.Scott et al [9] proposed load control method to tackle voltage rise issues due to embedded generation.

Voltage / reactive power control task is an optimization problem. T.Niknam et al [10] proposed volt/var control in the distribution system with DG using conventional GA. In this work single objective function has been considered and parameter tuning is the main drawback of this approach. Batrinu et a [11] considered time varying loads and used nested evolutionary programming for solving voltage control problem in a distribution system. 
However, this approach requires high computational burden even for a small test system.

The key issues in volt/var control problem of a distribution system with DG includes:

a) Improvement of voltage profile: Since introduction of DG makes the voltage profile complicated and affects the power quality, deviation of the voltage in each node from a certain reference voltage is considered as an indicative to quantify voltage profile improvement. So minimization of the voltage deviation is considered as one of the objective functions.

b) Network loss reduction: DG causes reverse power flow in the network and alters the power flows thereby affecting the distribution system operation and economy. Hence minimization of the losses is also considered as objective function.

Minimization of voltage deviation alone cannot minimize the losses. Therefore both the objective functions are considered separately for minimization to formulate the proposed multiobjective optimization problem.

The values of the penalty or weighting factors depend on the importance of the objective functions and constraints. The values of the objective functions and constraints vary from network to network which causes weighting factors to vary from network to network as a result of which for every network weights are to be tuned. To overcome the above mentioned difficulties, in this work, a method based on NSGA [12] is proposed for solving the multi-objective volt/var control problem in the distribution system with DG.

\section{PROBLEM FORMULATION}

The volt/var control of a radial distribution network with dispersed generation is considered. For the volt/var control, step voltage regulator (SVR) with line rise compensation (LRC)/line drop compensation (LDC) function has been considered. The control parameters considered in this work are,

(1) Resistance of the LRC/LDC

(2) Reactance of the LRC/LDC

(3) Reactive power output of the DG

(4) Tap position of the regulator

\section{Objective Function}

A multi-objective optimization problem has been formulated with the following objective functions

\section{1) Minimization of the voltage deviation:}

Using load flow, voltages at all the buses are obtained. The difference between the voltage at each bus and the reference voltage is found to determine voltage deviation. Sum of all these individual voltage deviations are considered in forming the objective function.

$$
\operatorname{Min} f_{1}=\sum_{i=1}^{n}\left|V_{i}-V_{r e f}\right|^{2}
$$

Where $\mathrm{n}$ is the no. of nodes in the network considered, $\mathrm{V}_{\text {ref }}$ is the reference voltage and $v_{i}$ is the voltage at $\mathrm{i}^{\text {th }}$ bus.

\section{2) Minimization of the losses:}

Using load flow line losses in all the branches are determined using the relation,

$$
\begin{gathered}
S_{m n}=V_{m}^{*} I_{m} \quad S_{n m}=V_{n}^{*}\left(-I_{m}\right) \\
\\
\end{gathered}
$$

$\mathrm{m}=1,2 \ldots . \mathrm{b}$ (no. of branches) and $\mathrm{n}=\mathrm{m}+1$.

Where V and I are bus voltages and branch currents respectively. Sum of all these individual line losses are taken in forming the objective function.

$$
\operatorname{Min} f_{2}=\sum_{j=1}^{m} \operatorname{loss}_{j}
$$

Where $\mathrm{m}$ is the no. of branches in the network.

\section{3) Constraints}

a) Voltage constraint:

Bus voltages should be maintained within the permissible range. Using the load flow, the bus voltages are obtained and are compared with limiting values of voltage.

$$
v_{\min }<v_{i}<v_{\max }
$$

Where $v_{\min }$ is the minimum acceptable bus voltage, and $v_{\max }$ is the maximum acceptable bus voltage.

\section{b) Current constraint:}

Line currents should be maintained within the acceptable range. Using load flow, line currents are determined using the relation,

$$
I_{m}=-\left(J_{m+1}\right)+\sum I_{m+1}
$$


Where, $I_{m}$ is the current in $\mathrm{m}^{\text {th }}$ branch and $\mathrm{J}_{\mathrm{m}+1}$ is the current injected at node $m+1$ and $I_{m+1}$ is the currents in branches connected to node $m+1$. The nodal currents are calculated using,

$$
\begin{gathered}
J_{i}^{k}=\frac{S_{i}}{\left(V_{i}^{k-1}\right)^{*}} \\
\mathrm{i}=1,2 \ldots \ldots \mathrm{n} \text { (no. of nodes) }
\end{gathered}
$$

where $S_{i}$ and $V_{i}$ are the specified power and voltages at $i^{\text {th }}$ node. The line currents are compared with the limiting values of current.

$$
i_{\text {min }}<i_{m}<i_{\text {max }}
$$

Where $i_{\min }$ is the minimum acceptable line current, $i_{m}$ is the current in the $\mathrm{m}^{\text {th }}$ line and $i_{\max }$ is the maximum acceptable line current.

Since volt/ var control is considered, the improvement of voltage profile is the primary goal of this work, the objective function, minimization of the voltage deviation is given first preference and minimization of the losses is given the second preference.

Voltage limit of $\pm 5 \%$ has been considered in this study.

\section{NSGA BASED OPTIMAL VOLT/VAR CONTROL}

\subsection{Voltage Control using SVR with LRC/LDC}

Since the conventional voltage regulators cannot handle the reverse power flow and steep voltage fluctuations, new voltage regulation methods are to be considered to mitigate such problems. In this work, step voltage regulator (SVR) with line rise compensation (LRC) / line drop compensation (LDC) function voltage control method has been considered. SVRs are autotransformers with individual taps on their windings. LRC/LDC is a technique that has been used to mitigate the voltage rise/ voltage drop on a bus in a distribution network there by improving the voltage profile of distribution network buses with DG's connected to them or when the local load is high

\subsection{Non-Dominated Sorting Algorithm (NSGA)}

Genetic algorithm, which is one of the evolutionary algorithms, is a search and optimization tool, which works differently compared to classical search and optimization methods. Genetic algorithm approach is used due to its broad applicability, global perspective and high accuracy.

Non-dominated sorting genetic algorithm is one of the evolutionary algorithms and is a method for global search and optimization. NSGA is a powerful technique to solve multiobjective optimization problems. In the Non-dominated sorting method, a pareto-surface is found by sorting the individuals in the population. All the non-dominated individuals in the population are assigned with one fitness value (dummy). Then the rest of the population is sorted again. A pareto-surface is found and these individuals are assigned with another fitness value. This process is repeated until all the individuals receive fitness value. Then the fitness values are recalculated, so that the individuals on the first pareto-surface get maximum fitness value.

In this method the tuning of parameter is not needed to solve the multi-objective volt/var control problems. NSGA based approach is used due to its wide applicability, good accuracy and ease of use.

Considering the difficulty in tuning of parameters and advantages of NSGA, a method based on NSGA introduced by Deb [12] has been proposed for the voltage / reactive power control problem of a distribution system with DG.

\subsection{Scheme of NSGA based optimal volt / var control}

\subsubsection{Flow chart}

The flow chart of NSGA based volt/var control algorithm is given in Figure 1. The algorithm for optimal volt/var control is explained in the next section 3.3.2. 


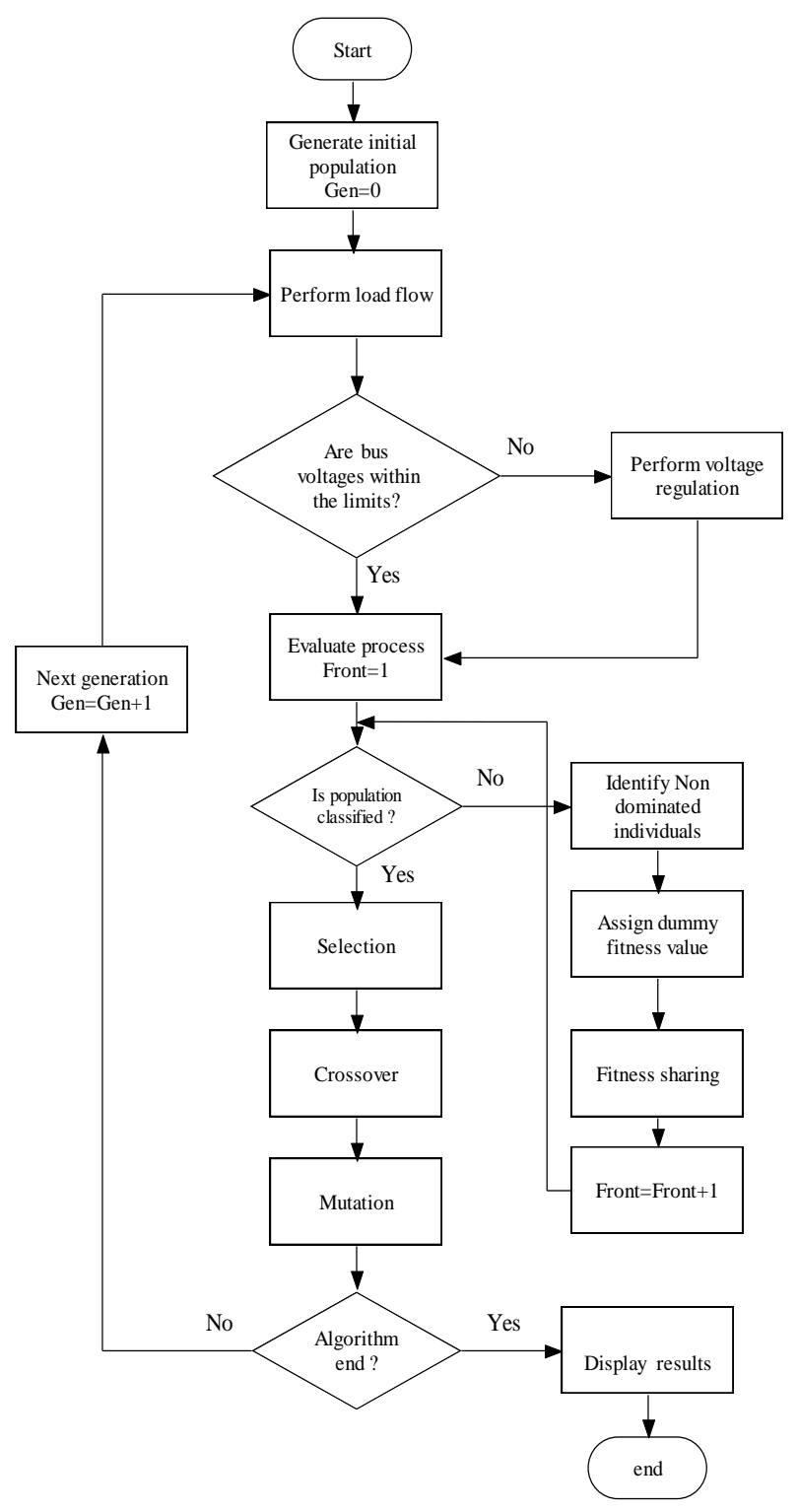

Figure 1. Flow chart of NSGA based volt/var control algorithm

\subsubsection{Algorithm}

\section{Step-1 Start}

Step-2 Initialize the population. Consider a set of $\mathrm{N}$ population members, each having $\mathrm{M}(\mathrm{M}>1)$ objective function values.

Step-3 Perform the load flow on the test radial distribution system with DG under consideration

Step-4 Identify the buses at which the voltages are out of statutory limits.
Step-5 Perform voltage regulation using SVRs with LRC/LDC functions located at appropriate places in the network

Step-6 Find non dominated set of solutions:

For a problem with multi- objective function, a solution $\mathrm{x}_{1}$ is said to dominate the other solution $\mathrm{x}_{2}$ if both the following conditions are true:

a) The solution $x_{1}$ is no worse than $x_{2}$ in all objectives

b) The solution $\mathrm{x}_{1}$ is strictly better than $\mathrm{x}_{2}$ in at least one objective.

Step-6.1 Set $n=1$

Step-6.2 For all $\mathrm{m}=1 \ldots . . \mathrm{N}$ and $\mathrm{m} \# \mathrm{n}$, for all $\mathrm{M}$ objectives compare solutions $\mathrm{x}_{\mathrm{n}}$ and $\mathrm{x}_{\mathrm{m}}$ for domination using the conditions.

Step-6.3 If for any $m, x_{n}$ is dominated by $x_{m}$, treat $x_{n}$ as dominated

Step-6.4 If all solutions in the set are considered i.e. when $n=N$ is reached, go to the Step-6.5, else increment $n$ by one and go to step6.2.

Step-6.5 All solutions that are not marked 'dominated' are 'non-dominated' solutions.

Step-6.6 All these non-dominated solutions are assumed to constitute the first non-dominated front in the population and assigned a large dummy fitness value. All these solutions, in this way, have an equal reproductive potential.

Step-6.7 These non-dominated solutions are then shared with their dummy fitness value in order to maintain population diversity. These non-dominated individuals are ignored temporarily to process in the same way the rest of the population members.

Step-7 Perform Selection operation according to dummy fitness values.

Step-8 Perform Crossover operation

Step-9 Perform Mutation operation

Step-10 Check for termination of the algorithm as per stopping criteria. If met display the results (i.e. optimal set points of voltage/reactive power control units considered as detailed in section 2) and stop or else increment generation count and go to step 3 .

Step-11 Stop 


\section{TEST SYSTEM AND SIMULATION RESULTS}

\subsection{Test System}

The test system considered for this work is shown in Figure 2 [4].The parameters of the test system are shown in Table 1.

Table 1. Test System data

\begin{tabular}{ll}
\hline Network data & 5 \\
No. of buses & 4 \\
No. of branches & $1.03 \mathrm{pu}$. \\
Voltage $\left(\mathrm{V}_{1}\right)$ & 4 \\
No. of load buses & 1 \\
No. of DGs & Bus no.3 \\
Location of DG & \\
DG details: & $20 \mathrm{MW}$ \\
P & 0.9 lagging \\
Pf & $9.686 \mathrm{MVAR}$ \\
$\mathrm{Q}$ & $100 \mathrm{MVA}$ \\
Base power $\mathrm{S}_{\text {base }}$ & $1.6 \mathrm{MW}, 1.2 \mathrm{MVAR}$ \\
Load size $\mathrm{P}_{\mathrm{L}} \mathrm{Q}_{\mathrm{L}}$ & $11 \mathrm{kV}$ \\
Network voltage & \\
\hline
\end{tabular}

\subsection{Simulation Results}

The proposed methodology has been applied on the distribution system shown in Figure 2. The control variables considered are as listed in Section 2. The multi-objective function considered for this work is discussed in Section 2. The simulation has been carried out using MATLAB.

Tuning of the parameters is the key issue in volt/var control optimization problems. Since the values of weight factors vary from network to network, proper tuning is difficult and if the weights are not properly assigned the solution obtained may not be the optimal one.

To alleviate the problem of tuning of the weights a method based on NSGA has been proposed to solve the multi-objective volt/var control problem. The scheme of the proposed method is discussed in Section 3. The value of the fitness function plotted against generations using the proposed method is shown in Figure 3. The optimal solution is obtained in less than 7 generations. Generation wise variation of different objective functions is plotted in Figure 4. It is evident from the Figure 4 that the constraints are satisfied and the solution obtained is the optimal one.

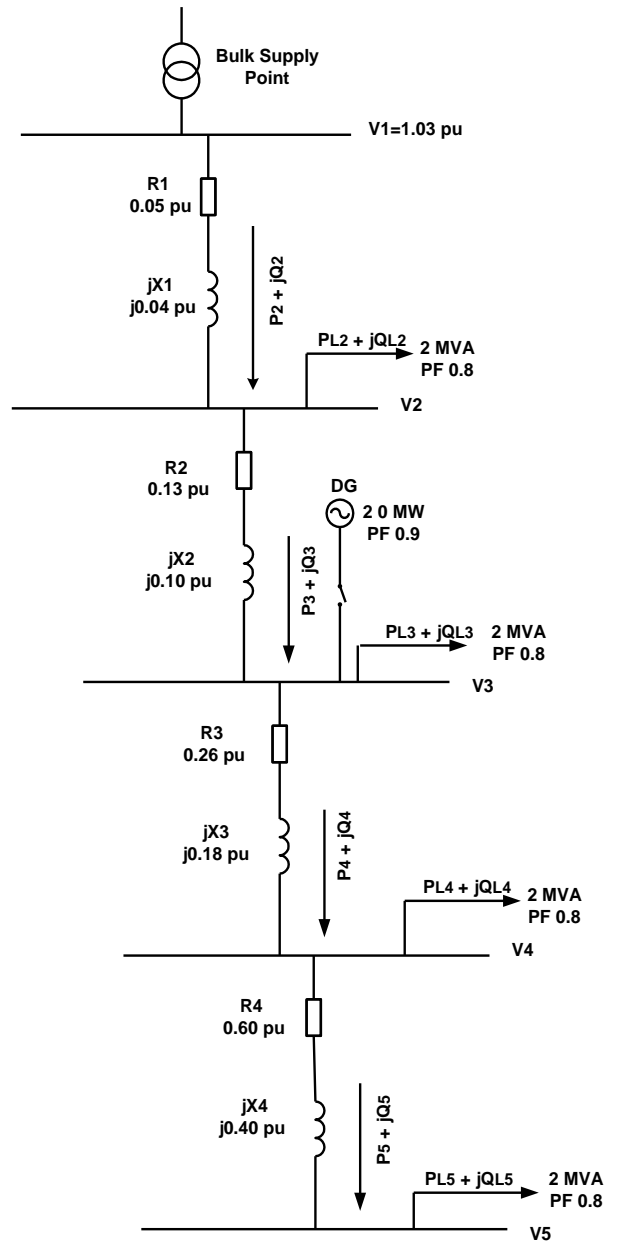

Figure 2. Test Radial Distribution Network

Voltage profile of the test system with the proposed method is plotted in Figure 5. It is clear that with this method, the voltage profile has improved to a very large extent and the voltages at all the buses are well within the statutory limits. Generation wise variation of the value of fitness function with the proposed approach and by assigning penalty factors i.e. simple genetic algorithm approach (SGA) are compared in Figure 6. It can be noted from Figure 6 that the proposed method takes less no. of generations to converge than the other approach.

The values of the objective functions without voltage regulation, with the voltage regulation prior to optimization, optimization using weighting factors (SGA) and optimization using proposed method are compared in Table 2.

It can be observed that with the proposed method, values of the objective functions are optimal. It is also observed that, when the multi-objective functions are considered, the losses are less compared to the objective function with voltage deviation alone and with out losses is considered. 


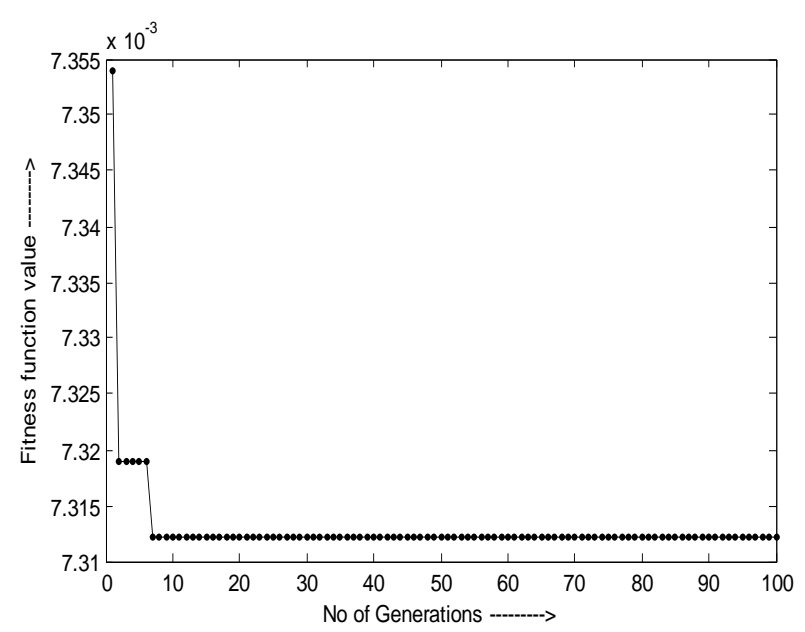

Figure 3. Fitness function vs. Generation

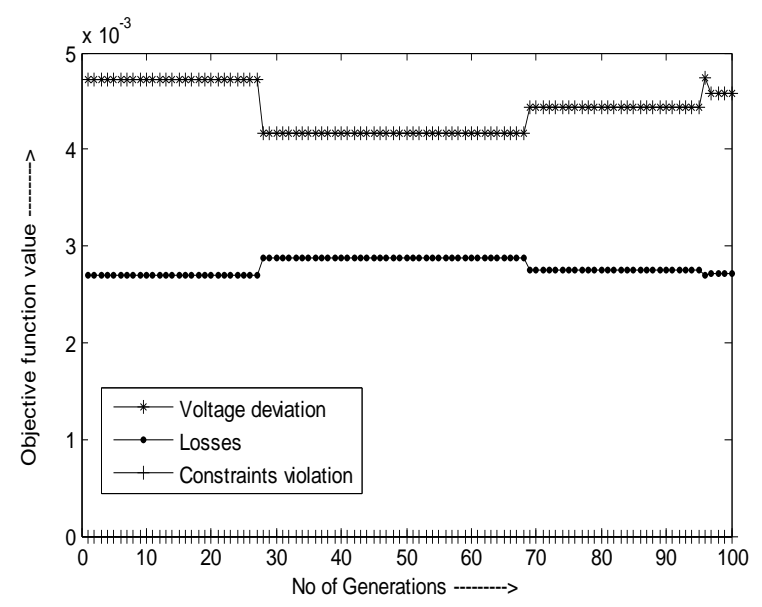

Figure 4. Variation of the Objective functions vs. Generation

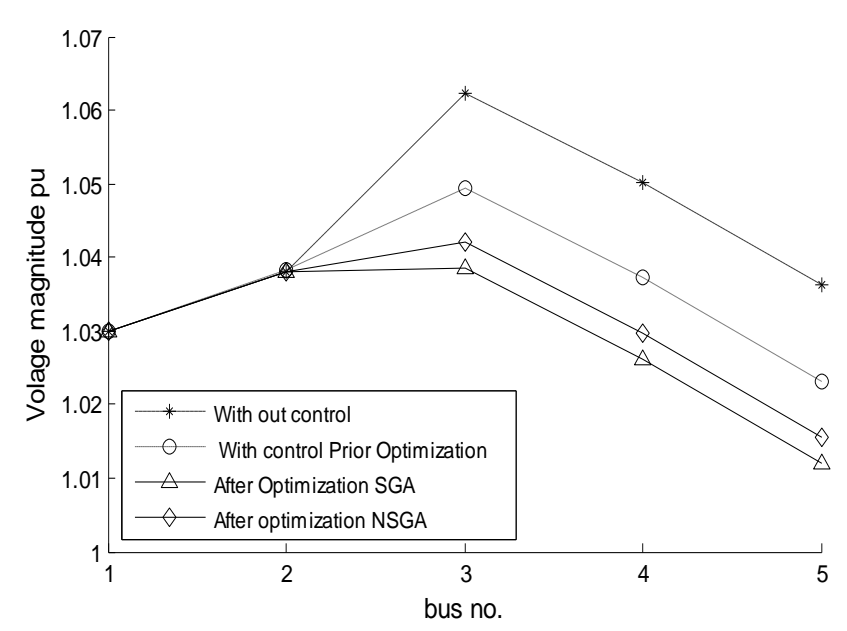

Figure 5. Voltage profile of the test distribution system

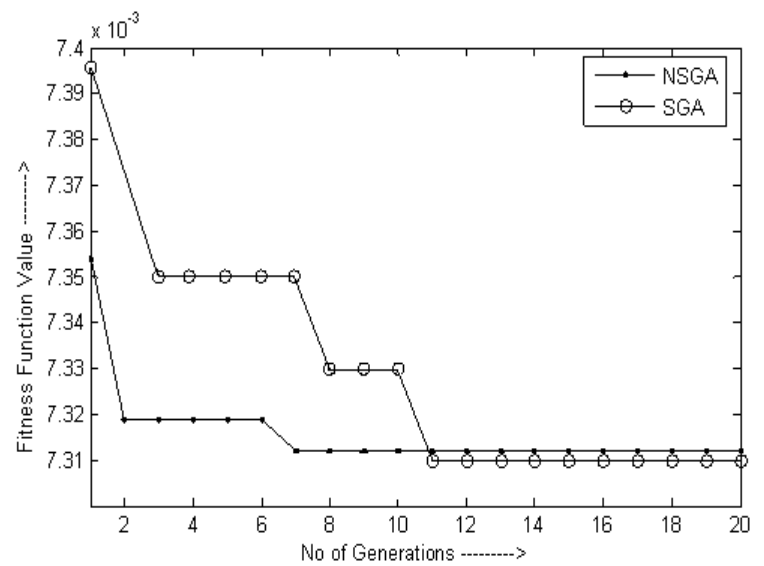

Figure 6. Comparison of two methods

Table 2. Values of Objective functions

EVoltage deviation Total Losses

pu $\quad k V A$

Without control

0.0101

576.1

Prior to optimization

0.0091

537.1

SGA

0.0052

310.0

Proposed method

0.0046

270.0

With only Voltage

0.0044

deviation

\section{CONCLUSIONS}

In this paper, a method based on NSGA has been proposed to solve the multi-objective volt/var control problem of a distribution system with DG. The proposed method has been implemented for finding the optimal set points of volt/var controls in the distribution system with dispersed generation. This technique does not need the parameter tuning to solve the multiobjective volt/var control problems. The method proposed is found to be simple, accurate and easy to apply. This technique is a direct approach to solve volt/var control problems and takes very few numbers of iterations to converge. So, the proposed method is very much suitable to solve multi-objective volt/var control optimization problem of a distribution system with DG which is very important in the area of power system operation and control and also a prominent step towards distribution system automation.

\section{REFERENCES}

[1] C.L. Masters, "Voltage rise: the big issue when connecting embedded generation to long $11 \mathrm{kV}$ overhead lines", IEE 
Power Engineering J., vol. 16, no.1, pp. 5-12, February 2002.

[2] "IEEE standard for interconnecting distributed resources with electric power systems" IEEE standard $1547^{\mathrm{TM}}-2003$.

[3] K.Tran and M.Vaziri, "Effects of dispersed generation (DG) on distribution systems", Proc. of IEEE Power Engineering Society General Meeting, vol. 3, pp.2173-2178, 2005.

[4] A.E. Kiprakis and A.R Wallace, "Maximizing energy capture from distributed generators in weak networks", IEE Proceedings on Generation Transmission and Distribution, vol. 151, no. 5, pp. 611-618, September 2004.

[5] T-E Kim and J-E Kim, "Voltage regulation coordination of distributed generation system in distribution system",Proc. of IEEE Power Engineering Society Summer Meeting, vol. 1, pp.480-484, 2001.

[6] T. Gonen, "Electric power distribution system engineering", Second Edition, CRC Press, 2007.

[7] T-E Kim and J-E Kim, "Considerations for the feasible operating range of distributed generation interconnected to power distribution system", Proc. of IEEE Power Engineering Society Summer Meeting, vol. 1, pp.42-48, 2002.

[8] J-Ho Choi and J-Chul Kim, "Advanced voltage regulation method at the power distribution systems interconnected with dispersed storage and generation systems", IEEE Trans. Power Delivery, vol.15, no. 2, pp.691-696, April 2000.

[9] N.C. Scott, D.J. Atkinson and J. E. Morrell,'Use of load control to regulate voltage on distribution networks with embedded generation", IEEE Trans. Power Systems, vol. 17, no.2, pp. 510-515, May 2002.

[10] T. Niknam, A.M. Ranjbar and A.R. Shirani, "Impact of distributed generation on Volt/ Var control in distribution Networks" in Proc. IEEE Bologna Power Tech Conference, Italy,,vol. 3, June 23-26, 2003.

[11] F. Batrinu, E. Carpaneto, G. Chicco, M. De Donno, R. Napoli , P. Postolache, C. Toader and R. Porumb, "New nested evolutionary programming approach for voltage control optimization with distributed generation", in Proc. IEEE Mediterranean Electrotechnical Conference, MELECON, vol. 3, pp. 1007-1010, 2004.

[12] K. Deb," Multi-objective optimization using evolutionary algoritms", Newyork, John Wiley, 2001. 